\title{
Impaired neutralizing antibody response to COVID-19 mRNA vaccines in cancer patients
}

\author{
Cong Zeng ${ }^{1,2+}$, John P. Evans ${ }^{1,2,3 \dagger}$, Sarah Reisinger ${ }^{4 \dagger}$, Jennifer Woyach ${ }^{5}$, Christina Liscynesky ${ }^{6}$, \\ Zeinab El Boghdadly ${ }^{6}$, Mark P. Rubinstein ${ }^{7}$, Karthik Chakravarthy ${ }^{7}$, Linda Saif ${ }^{8,9,10}$, Eugene M. Oltz ${ }^{11}$, \\ Richard J. Gumina ${ }^{12}$, Peter G. Shields ${ }^{4^{*}}$, Zihai Li ${ }^{7^{*}}$ and Shan-Lu Liu ${ }^{1,2,10,11^{*}}$
}

\begin{abstract}
There is currently a critical need to determine the efficacy of SARS-CoV-2 vaccination for immunocompromised patients. In this study, we determined the neutralizing antibody response in 160 cancer patients diagnosed with chronic lymphocytic leukemia (CLL), lung cancer, breast cancer, and various non-Hodgkin's lymphomas (NHL), after they received two doses of mRNA vaccines. Serum from 46 mRNA vaccinated health care workers (HCWs) served as healthy controls. We discovered that (1) cancer patients exhibited reduced neutralizing antibody titer $\left(\mathrm{NT}_{50}\right) \mathrm{com}$ pared to HCWs; (2) CLL and NHL patients exhibited the lowest $\mathrm{NT}_{50}$ levels, with 50-60\% of them below the detection limit; (3) mean $N T_{50}$ levels in patients with CLL and NHL was $~ 2.6$ fold lower than those with solid tumors; and (4) cancer patients who received anti-B cell therapy exhibited significantly reduced $\mathrm{NT}_{50}$ levels. Our results demonstrate an urgent need for novel immunization strategies for cancer patients against SARS-CoV-2, particularly those with hematological cancers and those on anti-B cell therapies.
\end{abstract}

\section{Dear Editor,}

In response to the global public health crisis caused by the COVID-19 pandemic, several SARS-CoV-2 vaccines were rapidly developed including the Pfizer/BioNTech BNT162b2 and Moderna mRNA-1273 mRNA vaccines. However, clinical trials of these mRNA vaccines did not investigate their efficacy in vulnerable populations, including immunocompromised patients. With rising vaccination rates and an easing of public health measures, there is a critical need to determine the efficacy

\footnotetext{
*Correspondence: Peter.Shields@osumc.edu; Zihai.Li@osumc.edu; Liu.6244@osu.edu

${ }^{\dagger}$ Cong Zeng, John P. Evans and Sarah Reisinger contributed equally to this letter

${ }^{1}$ Center for Retrovirus Research, The Ohio State University, Columbus, OH 43210, USA

${ }^{4}$ Comprehensive Cancer Center, James Cancer Hospital, The Ohio State University, Columbus, OH 43210, USA

${ }^{7}$ Division of Medical Oncology, Department of Internal Medicine, Pelotonia Institute for Immuno-Oncology, The Ohio State University Comprehensive Cancer Center, Columbus, OH 43210, USA

Full list of author information is available at the end of the article
}

of SARS-CoV-2 vaccination for such patients, who may experience a reduced efficacy of administered vaccines [1]. It has already been demonstrated that organ transplant recipients, who are under immunosuppressive therapy to prevent rejection, exhibit reduced responsiveness to SARS-CoV-2 vaccination [2]. Cancer patients represent another critical population of immunocompromised individuals who, due to the nature of the disease or to treatment with immunomodulatory therapies, may not exhibit a robust response to mRNA vaccination. A better understanding of the factors governing response to vaccination in cancer patients is critical to inform clinical decisions about the need for booster doses, the timing of vaccine administration, the need to interrupt treatment courses for vaccination, and general guidance about the level of protection achieved by vaccination in cancer patients. To this end, this study examines the neutralizing antibody response to Pfizer/BioNTech BNT162b2 and Moderna mRNA-1273 vaccination in a cohort of patients with solid tumor and hematological malignancies.

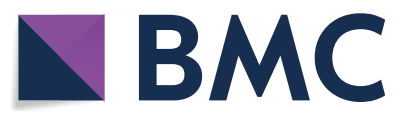

(c) The Author(s) 2021. Open Access This article is licensed under a Creative Commons Attribution 4.0 International License, which permits use, sharing, adaptation, distribution and reproduction in any medium or format, as long as you give appropriate credit to the original author(s) and the source, provide a link to the Creative Commons licence, and indicate if changes were made. The images or other third party material in this article are included in the article's Creative Commons licence, unless indicated otherwise in a credit line to the material. If material is not included in the article's Creative Commons licence and your intended use is not permitted by statutory regulation or exceeds the permitted use, you will need to obtain permission directly from the copyright holder. To view a copy of this licence, visit http://creativecommons.org/licenses/by/4.0/. The Creative Commons Public Domain Dedication waiver (http://creativeco mmons.org/publicdomain/zero/1.0/) applies to the data made available in this article, unless otherwise stated in a credit line to the data. 
The study population included 160 cancer patients (54 chronic lymphocytic leukemia (CLL), 45 non-Hodgkin's lymphoma (NHL), 29 lung cancer, 30 breast cancer, and 2 breast cancer with CLL) recruited through medical record screening for vaccine appointments or recent post-vaccine administration, as well as an independent cohort of 46 health care workers (HCWs), who have no history of cancer. Cancer patients had a median age of 66 years while the median age of HCWs was 38 years. No cancer patient or HCW was COVID-19 positive as confirmed by nucleocapsid-based ELISA. About $61 \%$ of cancer patients $(n=98)$ and $52 \%$ of the HCWs $(n=24)$ received BNT162b2, compared to $39 \%(\mathrm{n}=62)$ and $48 \%$ $(\mathrm{n}=22)$ who received the mRNA-1273, respectively. We collected serum samples for 159/160 cancer patients between 31 and 232 days (median 134days) post-second dose, and HCW serum samples were obtained at 6 months post-second dose. Cancer diagnoses and treatments of the patients are shown in Table 1 . The largest treatment groups were 47 patients with B-cell malignancies (28 CLL and 19 NHL) who received B cell depletion therapy or other B cell-suppressing drugs (such as antiCD20 monoclonal antibodies and Bruton tyrosine kinase (BTK) inhibitors) during the study period; and $46 \%$ $(n=28)$ of solid tumor patients received immune checkpoint inhibitors against PD-1 or PD-L1.

We assessed sera for neutralizing antibody titers using a secreted Gaussia-luciferase SARS-CoV-2-pseudotypedlentivirus neutralization assay as previously described (Additional file 1) [3]. Briefly, pseudotyped virus was incubated with serial dilutions of patient sera and used to infect HEK293T-ACE2 cells (BEI NR-52,511). Infected cells then secreted Gaussia-luciferase into the culture media which was harvested 48 and $72 \mathrm{~h}$ after infection, and luminescence was measured by a BioTek Cytation 5 plate-reader. The resulting luciferase output was used to calculate a neutralization titer at $50 \%$ efficiency of maximal inhibition $\left(\mathrm{NT}_{50}\right)$. To ensure valid comparisons, the

Table 1 Demographic information of cancer patients

\begin{tabular}{|c|c|c|c|c|c|c|}
\hline & \multicolumn{2}{|c|}{ Total $(n=160)$} & \multicolumn{2}{|c|}{ Male $(n=85)$} & \multicolumn{2}{|c|}{ Female $(n=75)$} \\
\hline & $n$ & $(\%)$ & $n$ & $(\%)$ & $n$ & $(\%)$ \\
\hline \multicolumn{7}{|l|}{ Age Group (years) } \\
\hline $30-44$ & 11 & 6.9 & 1 & 1.2 & 10 & 13.3 \\
\hline $45-59$ & 35 & 21.9 & 12 & 14.1 & 23 & 30.7 \\
\hline $60-74$ & 96 & 60.0 & 58 & 68.2 & 38 & 50.7 \\
\hline $75-85$ & 18 & 11.3 & 14 & 16.5 & 4 & 5.3 \\
\hline \multicolumn{7}{|l|}{ Race } \\
\hline African American/Black & 6 & 3.8 & 2 & 2.4 & 4 & 5.3 \\
\hline Asian Chinese & 3 & 1.9 & 2 & 2.4 & 1 & 1.3 \\
\hline Asian Japanese/White & 1 & 0.6 & 1 & 1.2 & 0 & 0.0 \\
\hline Other & 2 & 1.3 & 1 & 1.2 & 1 & 1.3 \\
\hline White & 148 & 92.5 & 79 & 92.9 & 69 & 92.0 \\
\hline \multicolumn{7}{|l|}{ Vaccine Type } \\
\hline Moderna & 62 & 38.8 & 34 & 40.0 & 28 & 37.3 \\
\hline Pfizer & 98 & 61.3 & 51 & 60.0 & 47 & 62.7 \\
\hline \multicolumn{7}{|l|}{ Cancer type } \\
\hline CLL & 54 & 33.8 & 40 & 47.1 & 14 & 18.7 \\
\hline Lung & 29 & 18.1 & 18 & 21.2 & 11 & 14.7 \\
\hline Breast & 30 & 18.8 & 0 & 0.0 & 30 & 40.0 \\
\hline CLL/Breast & 2 & 1.3 & 0 & 0.0 & 2 & 2.7 \\
\hline Non-Hodgkin's Lymphoma & 45 & 28.1 & 27 & 31.8 & 18 & 24.0 \\
\hline \multicolumn{7}{|l|}{ Anti-B cell therapy } \\
\hline CLL & 28 & 17.5 & 23 & 27.1 & 5 & 6.7 \\
\hline Non-Hodgkin's Lymphoma & 19 & 11.9 & 11 & 12.9 & 8 & 10.7 \\
\hline \multicolumn{7}{|l|}{ Anti-PD-1/PD-L1 } \\
\hline Lung & 26 & 16.3 & 17 & 20.0 & 9 & 12.0 \\
\hline Breast & 2 & 1.3 & 0 & 0.0 & 2 & 2.7 \\
\hline
\end{tabular}

The anti-B cell therapy drugs include Obinutuzumab, Rituximab, Ibrutinib, Zanubrutinib, Pirtobrutinib and Acalabrutinib

The anti-PD-1/PD-L1 drugs include Nivolumab, Pembrolizumab, Durvalumab and Atezolizumab 
serum samples of all cancer patients and HCWs were processed side-by-side in the same experiment.

We first compared the neutralizing antibody titers of cancer patients with those of HCWs. Overall, cancer patients exhibited reduced neutralizing antibody responses, with a mean $\mathrm{NT}_{50}$ of 220 compared to a mean $\mathrm{NT}_{50}$ of 522 for HCWs (Fig. 1a); this is despite the relatively shorter median time (134days) after the second dose of vaccination for cancer patients as compared to HCWs, which is an average of $\sim 180$ days. Patients with CLL exhibited the lowest neutralizing antibody response, with over $61 \%(n=34)$ of patients exhibiting undetectable $\mathrm{NT}_{50}$ values (below 40), compared to $49 \%$, $31 \%$, and $28 \%$ for NHL $(n=22)$, lung cancer $(n=9)$, and breast cancer patients $(n=9)$, respectively (Fig. $1 b)$. The mean $\mathrm{NT}_{50}$ of patients with CLL and NHL (158 and 127, respectively) was $\sim 2.6$ fold lower than that of solid tumor patients (369) (Fig. 1a). Interestingly, there were a few CLL patients that exhibited high titer while none were observed for the NHL patients (Fig. 1b).

Given the common usage of B-cell depleting therapies in the treatment of hematological cancers and their likelihood of impacting vaccine efficacy, we then examined the effect of anti-B-cell therapy on neutralizing antibody response. The treatment included anti-CD20 antibodies Obinutuzumab and Rituximab, as well as BTK inhibitors Ibrutinib, Zanubrutinib, Pirtobrutinib, and Acalabrutinib. Notably, we found that CLL and NHL patients who received anti-B cell therapy exhibited 2.7-fold $(\mathrm{p}=0.0483)$ and 3.1-fold $(\mathrm{p}=0.0030)$ reduced neutralizing antibody response to mRNA vaccine compared to those without anti-B cell therapy, respectively (Fig. 1c).

The programmed death-1 (PD-1) receptor is an important immune checkpoint molecule that promotes exhaustion/dysfunction in chronically activated T-cells. Disruption of PD-1 or its ligand PD-L1 is a common treatment to rejuvenate $\mathrm{T}$ cell function in cancer patients [4]. Given this role, we examined how anti-PD-1/PD-L1 treatments might modulate the host immune response to mRNA vaccination. However, we did not find significant differences in $\mathrm{NT}_{50}$ or development of immune-related adverse events between anti-PD-1/PD-L1 antibodytreated and un-treated lung/breast cancer patients (Fig. 1d).

Other factors potentially impacting immune stimulation were also assessed, including age and gender of patients, types of vaccine received and time of sample collection. Moderna mRNA-1273 outperformed Pfizer BNT162b2 vaccine in mean $\mathrm{NT}_{50}$ by 2.8 -fold for HCWs $(\mathrm{p}=0.0053)$ and 2.1 -fold for cancer patients $(\mathrm{p}=0.0044)$ (Fig. 1e). This is consistent with our previous findings that Moderna mRNA-1273 vaccinated individuals exhibit higher $\mathrm{NT}_{50}$ levels compared to Pfizer BNT162b2 [5]. Given previous findings that neutralizing antibody response to mRNA vaccination is age dependent [6], we also examined the possible correlation between age and $\mathrm{NT}_{50}$ titer. However, no significant correlation between age and $\mathrm{NT}_{50}$ values was observed in these cancer patients (Fig. 1f). Notably, while male patients have been shown to exhibit higher $\mathrm{NT}_{50}$ levels following COVID19 disease [7], we found here that female patients in fact exhibited a higher level of virus neutralization with a mean $\mathrm{NT}_{50}$ of 299 compared to 154 for males ( $\mathrm{p}=0.0116$; Fig. 1g). This likely reflects an overrepresentation of older patients and patients with hematological cancers in males in our cohort (Table 1).

Given increasing concerns about declining efficacy of SARS-CoV-2 vaccines [8], we also examined the correlation between $\mathrm{NT}_{50}$ and time post second vaccine dose for these cancer patients. Indeed, we observed a significant, negative correlation $(\mathrm{p}=0.0194)$ between time after second dose of mRNA vaccination and $\mathrm{NT}_{50}$ value (Fig. $1 \mathrm{~h}$ ). These results confirm the waning immune protection of neutralizing antibodies that are conferred by mRNA vaccination.

In summary, by using a sensitive high-throughput lentivirus-based SARS-CoV-2 neutralization assay [3], we have examined the neutralizing antibody response of 160 cancer patients and compared, side by side, with that of 46 healthy HCWs. We observed about an approximately

\footnotetext{
(See figure on next page.)

Fig. 1 Neutralization of SARS-CoV-2 spike-pseudotyped lentivirus by sera of cancer patients and health care workers. a Comparison of 50\% neutralization titer $\left(\mathrm{NT}_{50}\right)$ between cancer patients and health care workers (HCWs). Serially diluted sera were incubated with SARS-CoV-2 spike-pseudotyped lentivirus, followed by infection of HEK293T-ACE2 cells. The assay was carried out side by side for samples of healthy individuals and cancer patients to ensure valid comparisons. b Distribution ranges of $\mathrm{NT}_{50}$ among four cancer patient groups. Note that 2 patients who had both CLL and breast cancer were included in each group. c Comparison of $\mathrm{NT}_{50}$ between anti-B cell therapy and no anti-B cell therapy in cancer patients. Twenty-eight out of the $54 \mathrm{CLL}$ patients and 19 out of the $45 \mathrm{NHL}$ patients received anti-B cell therapy, with drugs including BTK inhibitors and anti-CD20 monoclonal antibodies. $\mathbf{d}$ Comparison of $\mathrm{NT}_{50}$ between anti-PD1/PD-L1 and no anti-PD1/PD-L1 treatment in lung and breast cancer patients. e Comparison of $\mathrm{NT}_{50}$ between Moderna and Pfizer vaccinees in health care workers (HCWs) and cancer patients. $\mathbf{f}$ Correlative analysis between $\mathrm{NT}_{50}$ values and ages of cancer patients. $\mathbf{g}$ Comparison of $\mathrm{NT}_{50}$ values between male and female cancer patients. $\mathbf{h}$ Correlative analysis between $\mathrm{NT}_{50}$ values and days of collection after the second dose of vaccination. All correlative analyses were performed using Prism 5 (f and h). In all cases, $\mathrm{NT}_{50}$ values indicated at top were calculated by taking the inverse of the $50 \%$ inhibitory dilution values obtained from least squares regression non-linear curve modeled with Prism. Statistical significance was determined by a one-tailed unpaired t-test. CLL: Chronic Lymphocytic Leukemia; NHL: Non-Hodgkin's Lymphoma
} 


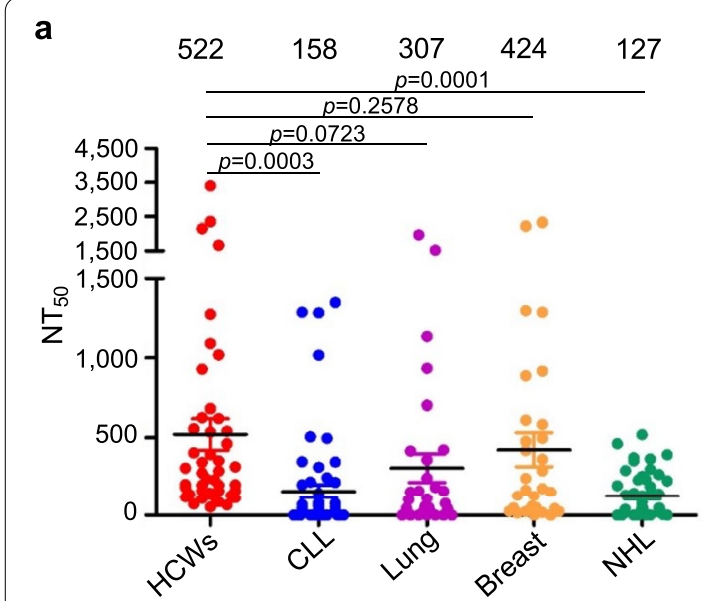

b

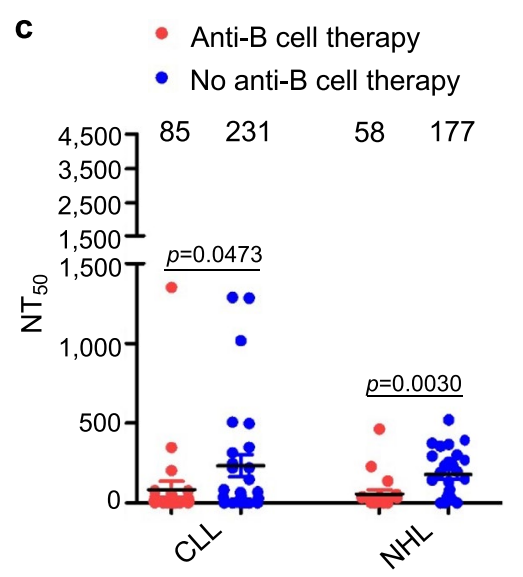

d

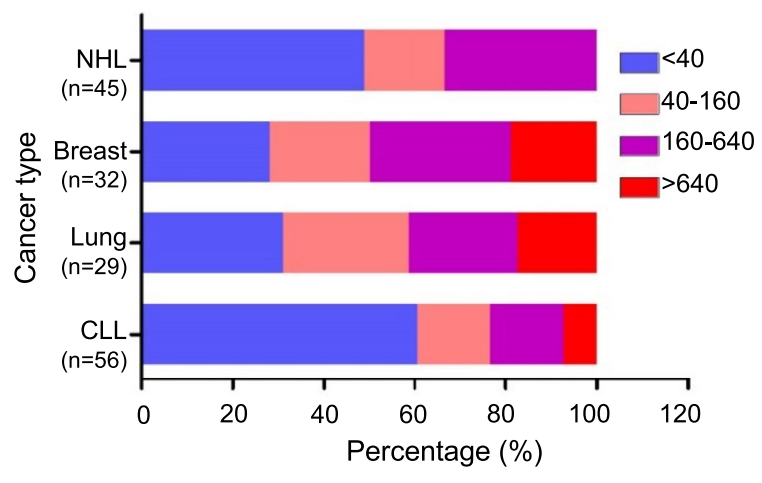

d Lung and Breast
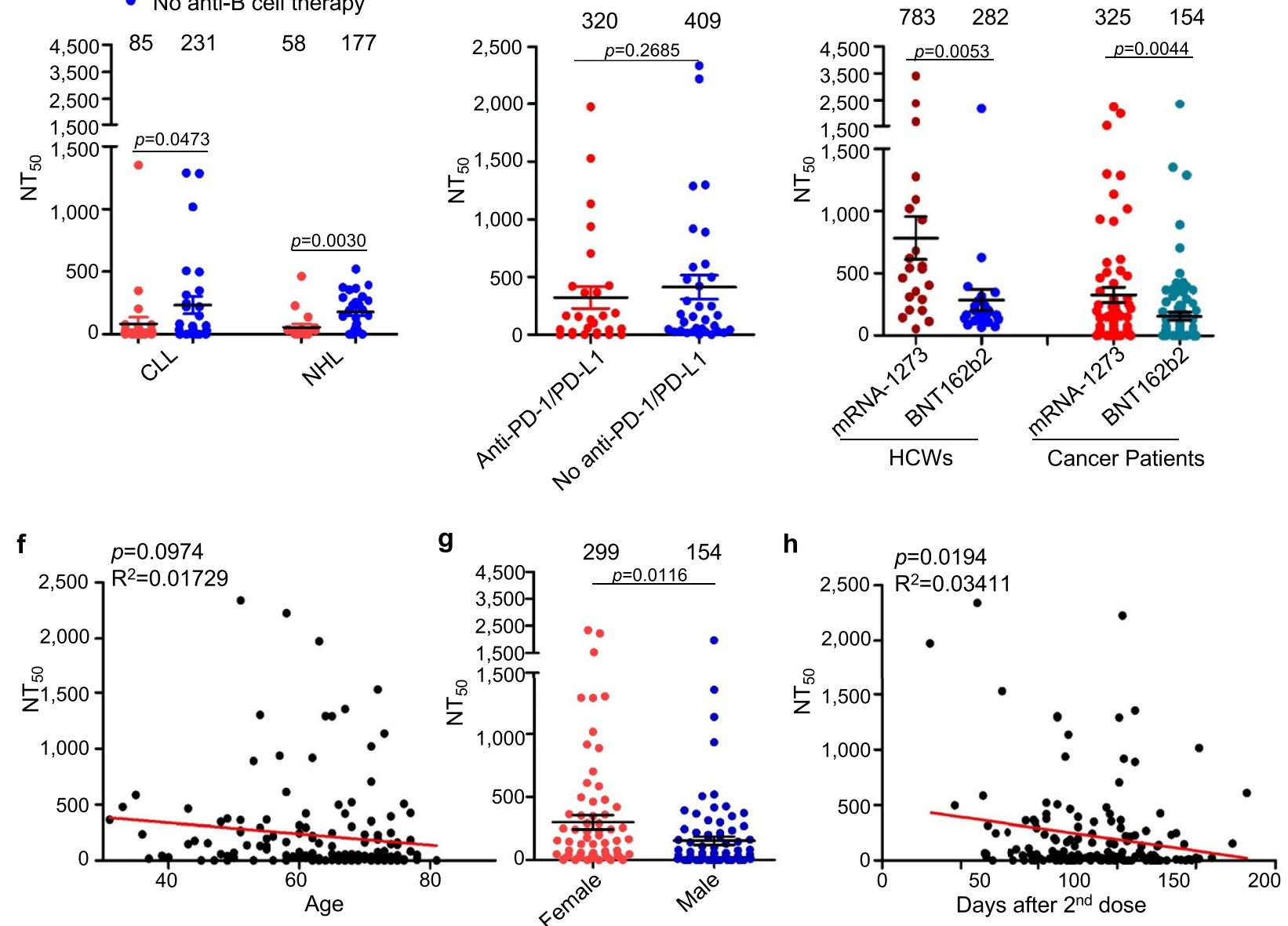

Fig. 1 (See legend on previous page.)

2.4-fold lower neutralizing antibody response in the cancer patients as compared to HCWs, following Pfizer/ BioNTech BNT162b2 or Moderna mRNA-1273 vaccination, clearly demonstrating a reduced efficacy of
SARS-CoV-2 spike antibody production among cancer patients. This, along with similar observations of some recent complementary studies [9], should inform the development of novel immunization strategies for cancer 
patients. In particular, we find that patients with hematological cancers, such as CLL and NHL, are least likely to respond to mRNA vaccination, with $50-60 \%$ of these patients showing no detectable levels of neutralizing antibody against the SARS-CoV-2 spike. Given these findings, booster vaccines may be of particular importance for these groups of cancer patients, with some studies already underway [10]. Additionally, our finding that B cell depletion or suppression drug treatment significantly reduced the neutralizing antibody response to mRNA vaccines may indicate a need for immunization to occur during disruptions or suspensions in specific treatment protocols.

Finally, to better protect immunocompromised populations with increased risk to COVID-19, we must further investigate the duration of vaccine induced immunity as well as the efficacy of booster vaccine doses to determine how to maintain protective immunity in this patient population. Additionally, further study on quality and durability of antigen-specific $\mathrm{T}$ and memory $\mathrm{B}$ cell responses will provide a more comprehensive understanding of the immune response to SARS-CoV-2 vaccination in these immunocompromised groups. It is also critical to determine the impact of specific treatment protocols on vaccine induced immunity and immunity duration to better inform clinical decisions about the time of vaccination or boosting and the potential need for disruptions in treatment protocols. Results from this work provide critical virological and immunological information for protecting vulnerable populations (Additional file 1).

\section{Abbreviations}

CLL: Chronic lymphocytic leukemia; NHL: Non-Hodgkin's lymphomas; HCW: Health care worker; $\mathrm{NT}_{50}$ : The 50\% neutralizing antibody titer; COVID-19: Coronavirus disease 2019.

\section{Supplementary Information}

The online version contains supplementary material available at https://doi. org/10.1186/s13578-021-00713-2.

Additional file 1. Materials and methods.

\section{Acknowledgements}

We thank members of Liu lab for sharing reagents and discussion. We also thank Donna Bucci, Jamie Hamon, Chelsea Bolyard, Kevin Weller, and Taylor Chatlos from the Immune Monitoring and Discovery Platform of the Pelotonia Institute for Immuno-Oncology for assistance in sample collection and discussion. We thank the Clinical Research Center/Center for Clinical Research Management of The Ohio State University Wexner Medical Center and The Ohio State University College of Medicine in Columbus, $\mathrm{OH}$, specifically Francesca Madiai, Claire Carlin, Dina McGowan, Breona Edwards, and Trina Wemlinger, for collection, processing, and management of samples through the Cardiovascular Medicine JB Biorepository. We thank the NIH AIDS Reagent Program and BEl Resources for supplying important reagents that made this work possible.

\section{Authors' contributions}

C.Z. performed virus neutralization assays, provided data analysis, contributed to manuscript drafting and editing. J.P.E. aided in neutralization assays as well as manuscript drafting and editing. S.R. was involved in study design and implementation, data collection, as well as review and editing of the manuscript. C.L. and Z.E.B. aided in study design. R.J.G. provided control HCW samples and contributed to review and editing of the manuscript. Z.L. contributed to the sample collection, sample processing, data collection and analysis. S.-L.L., Z.L., and P.G.S. contributed to the inception and design of the study; study administration and oversite; acquisition of funding and other resources; supervised research staff; drafting, review, and editing of the manuscript. J.W., Z.E.B., M.P.R., L.S., E.M.O., and K.C. provided thoughtful input as well as review and editing of the manuscript. S.-L.L. is the lead contact corresponding author and shall be contacted for reagents. All authors read and approved the final manuscript.

\section{Funding}

This work was supported by a fund provided by an anonymous private donor to The Ohio State University and NCI U54CA260582 to S.-L.L. The content is solely the authors' responsibility and does not necessarily represent the official views of the NIH. S.-L.L. was additionally supported in part by NIH R01 Al150473. Z.L. was supported by The Ohio State University Comprehensive Cancer Center Cancer Center Support Grant (P30CA016058), and multiple NIH grants (R01 DK105033; R01 Al077283; R01 CA213290; P01 CA186866; R01 CA255334; P30 CA016058). P.G.S. was supported by multiple NIH grants including NCI P30 CA016058. S.R. and Z.L. were supported by the Peletonia Institute for Immune-Oncology. R.J.G. was supported by NIH R01 HL12744201A1, NIH U54CA260582, and the Cardiovascular Medicine JB Biorepository, and the Robert J. Anthony Fund for Cardiovascular Research. L.S. and E.M.O. were also supported by NIH U54CA260582. The Pelotonia Institute for Immuno-Oncology (P\|O) and the Immune Monitoring and Discovery Platform (IMDP) are funded in part by the Pelotonia community and the Ohio State University Comprehensive Cancer Center (P30CA016058).

Availability of data and materials

All data and materials will be made available upon requests.

\section{Declarations}

Ethics approval and consent to participate

The study was carried out with the consent approval from participants under approved IRB protocols 2021 C0041, 2020H0228 and 2020H0527 at The Ohio State University.

\section{Consent for publication}

All coauthors have agreed to publish the results of this study.

\section{Competing interests}

The authors report no competing interests related to this work.

\section{Author details}

${ }^{1}$ Center for Retrovirus Research, The Ohio State University, Columbus, $\mathrm{OH}$ 43210, USA. ²Department of Veterinary Biosciences, The Ohio State University, Columbus, OH 43210, USA. ${ }^{3}$ Molecular, Cellular and Developmental Biology Program, The Ohio State University, 43210 Columbus, OH, USA. ${ }^{4}$ Comprehensive Cancer Center, James Cancer Hospital, The Ohio State University, Columbus, $\mathrm{OH} 43210$, USA. ${ }^{5}$ Division of Hematology, James Comprehensive Cancer Center, The Ohio State University, Columbus, OH 43210, USA. ${ }^{6}$ Internal Medicine, Division of Infectious Diseases, Department of Internal Medicine, The Ohio State University, Columbus, $\mathrm{OH} 43210$, USA. ${ }^{7}$ Division of Medical Oncology, Department of Internal Medicine, Pelotonia Institute for Immuno-Oncology, The Ohio State University Comprehensive Cancer Center, Columbus, OH 43210, USA. ${ }^{8}$ Center for Food Animal Health, Animal Sciences Department, OARDC, College of Food, Agricultural and Environmental Sciences, Wooster, $\mathrm{OH}$ 44691, USA. ${ }^{9}$ Veterinary Preventive Medicine Department, College of Veterinary Medicine, The Ohio State University, 44691 Wooster, OH, USA.

${ }^{10}$ Viruses and Emerging Pathogens Program, Infectious Diseases Institute, The Ohio State University, Columbus, OH 43210, USA. ${ }^{11}$ Department of Microbial Infection and Immunity, The Ohio State University, 43210 Columbus, OH, USA. 
${ }^{12}$ Department of Medicine, The Ohio State University, Columbus, $\mathrm{OH} 43210$, USA.

Received: 5 November 2021 Accepted: 13 November 2021

Published online: 21 November 2021

\section{References}

1. Romano E, Pascolo S, Ott P. Implications of mRNA-based SARS-CoV-2 vaccination for cancer patients. J Immunother Cancer. 2021;9(6).

2. Rabinowich L, Grupper A, Baruch R, Ben-Yehoyada M, Halperin T, Turner D, Katchman E, Levi S, Houri I, Lubezky N. Low immunogenicity to SARS-CoV-2 vaccination among liver transplant recipients. J Hepatol. 2021;75(2):435-8.

3. Zeng C, Evans JP, Pearson R, Qu P, Zheng YM, Robinson RT, Hall-Stoodley L, Yount J, Pannu S, Mallampalli RK. Neutralizing antibody against SARSCoV-2 spike in COVID-19 patients, health care workers, and convalescent plasma donors. JCl insight. 2020;5(22).

4. Chamoto K, Hatae R, Honjo T. Current issues and perspectives in PD-1 blockade cancer immunotherapy. Int J Clin Oncol. 2020;25(5):790-800.

5. Zeng C, Evans JP, Faraone JN, Qu P, Zheng Y-M, Saif L, Otlz E, Lozanski G, Gumina RJ, Liu S-L. Neutralization of SARS-CoV-2 Variants of Concern Harboring Q. Mbio. 2021;677(5):e02510-02521.
6. Collier DA, Ferreira IA, Kotagiri P, Datir RP, Lim EY, Touizer E, Meng B, Abdullahi A, Elmer A, Kingston N. Age-related immune response heterogeneity to SARS-CoV-2 vaccine BNT162b2. Nature. 2021;596(7872):417-22.

7. Shrock E, Fujimura E, Kula T, Timms RT, Lee IH, Leng Y, Robinson ML, Sie BM, Li MZ, Chen Y. Viral epitope profiling of COVID-19 patients reveals cross-reactivity and correlates of severity. Science. 2020;370(6520):eabd4250

8. Keehner J, Horton LE, Binkin NJ, Laurent LC, Pride D, Longhurst CA, Abeles SR, Torriani FJ. Resurgence of SARS-CoV-2 infection in a highly vaccinated health system workforce. N Engl J Med. 2021;385(14):1330-2.

9. Greenberger LM, Saltzman LA, Senefeld JW, Johnson PW, DeGennaro LJ, Nichols GL. Antibody response to SARS-CoV-2 vaccines in patients with hematologic malignancies. Cancer Cell. 2021;39(8):1031-3.

10. Greenberger LM, Saltzman LA, Senefeld JW, Johnson PW, DeGennaro LJ, Nichols GL. Anti-spike antibody response to SARS-CoV-2 booster vaccination in patients with $B$ cell-derived hematologic malignancies. Cancer cell. 2021;39(8):1031-7.

\section{Publisher's Note}

Springer Nature remains neutral with regard to jurisdictional claims in published maps and institutional affiliations.
Ready to submit your research? Choose BMC and benefit from:

- fast, convenient online submission

- thorough peer review by experienced researchers in your field

- rapid publication on acceptance

- support for research data, including large and complex data types

- gold Open Access which fosters wider collaboration and increased citations

- maximum visibility for your research: over 100M website views per year

At BMC, research is always in progress.

Learn more biomedcentral.com/submissions 